\title{
A Linear Time Algorithm for Constructing Maximally Symmetric Straight-Line Drawings of Planar Graphs ${ }^{\star}$
}

\author{
Seok-Hee Hong and Peter Eades \\ National ICT Australia; School of Information Technologies, \\ University of Sydney, Australia \\ \{shhong, peter\}@it.usyd.edu.au
}

\begin{abstract}
This paper presents a linear time algorithm for constructing maximally symmetric straight-line drawings of biconnected and oneconnected planar graphs.
\end{abstract}

\section{Introduction}

Symmetry is one of the most important aesthetic criteria that clearly reveals the structure and properties of a graph. Symmetric drawings of a graph $G$ are clearly related to the automorphisms of $G$, and algorithms for constructing symmetric drawings have two steps:

1. Find the geometric automorphisms [3], and

2. Draw the graph displaying these automorphisms as symmetries.

This paper presents a linear time algorithm for constructing maximally symmetric straight-line drawings of biconnected and one-connected planar graphs. The first polynomial time algorithm which runs in quadratic time has appeared [4]. Here we present a linear time algorithm. A linear time algorithm for triconnected planar graphs [6] and disconnected graphs are dealt with in [9]. The following theorem summarizes our main result.

Theorem 1. There is a linear time algorithm that constructs maximally symmetric planar drawings of biconnected and one-connected planar graphs, with straight line edges.

In the next section, we review necessary background. In Section 3 and Section 4, we present a linear time algorithm for finding maximum number of symmetries (planar automorphisms) of biconnected and one-connected planar graphs. In Section 5, we describe the symmetric drawing algorithms.

\footnotetext{
* This research was partially supported by a grant from the Australian Research Council. For the full version of this paper, see $[7,8]$. National ICT Australia is funded by the Australian Government's Backing Australia's Ability initiative, in part through the Australian Research Council.
} 


\section{Symmetries and Planar Automorphisms}

An automorphism of a graph is a permutation of the vertex set that preserves adjacency. Symmetry in graph drawing is closely related to automorphisms of graphs: a symmetry of a graph drawing induces an automorphism of the graph. In this case, we say that the drawing displays the automorphism, and the automorphism is geometric [3]. Note that not every automorphism is geometric.

An automorphism $\alpha$ of a graph $G$ is a planar automorphism if there is a planar drawing $D$ of $G$ which displays $\alpha$. Note that not every geometric automorphism is planar. Further, the product of two planar automorphisms is not necessarily planar (because they may be displayed by different drawings). An automorphism group $A$ of a graph is a planar automorphism group if there is a single planar drawing of the graph that displays every element of $A[6]$. The central problem of this paper is to find a planar automorphism group of maximum size.

Planar Automorphism Problem

Input: A planar graph $G$.

Output: A maximum size planar automorphism group $A$ of $G$.

Previous research on the Planar Automorphism Problem has concentrated on subclasses of planar graphs $[5,11]$. Our aim is to give a linear time algorithm for planar graphs in general. We use connectivity to divide the problem into cases.

1. Triconnected graphs: a linear time algorithm is presented in [6].

2. Biconnected graphs: this is the topic of this paper.

3. One-connected graphs: this is the topic of this paper.

4. Disconnected graphs: a linear time algorithm is presented in [9].

Note that each case relies on the result of the previous case. The triconnected case was solved in [6]; the algorithm finds a plane embedding of $G$ that has maximum size planar automorphism group. Generators of the planar automorphism group of $G$ with given plane embedding are then derived. They also give a linear time drawing algorithm.

\section{The Biconnected Case}

If the input graph $G$ is biconnected, then we break it into triconnected components in a way that is suitable for the task. The overall algorithm is composed of three steps.

\section{Algorithm Biconnected_Planar}

1. Construct the SPQR-tree $T_{1}$ of $G$, and root $T_{1}$ at its center.

2. Reduction: For each level $i$ of $T_{1}$ (from the lowest level to the root level)

(a) For each leaf node on level $i$, compute labels.

(b) For each leaf node on level $i$, label the corresponding virtual edge of the parent node with the labels.

(c) Remove the leaf nodes on level $i$.

3. Compute a maximum size planar automorphism group at the labeled center. 
We briefly describe each step of the algorithm. The first step is to construct the SPQR-tree for the input biconnected planar graph. The SPQR-tree represents a decomposition of a biconnected planar graph into triconnected components. There are four types of nodes in the SPQR-tree $T_{1}$ and each node $v$ in $T_{1}$ is associated with a graph which is called as the skeleton of $v(\operatorname{skeleton}(v))$. The node types and their skeletons are:

1. $Q$-node: The skeleton consists of two vertices which are connected by two multiple edges.

2. $S$-node: The skeleton is a simple cycle with at least 3 vertices.

3. P-node: The skeleton consists of two vertices connected by at least 3 edges.

4. $R$-node: The skeleton is a triconnected graph with at least 4 vertices.

In fact, we use slightly different version of the SPQR-tree. We use the SPQR-tree without $Q$ nodes. The SPQR-tree is unique for each biconnected planar graph. Let $v$ be a node in $T_{1}$ and $u$ a parent node of $v$. The graph skeleton $(u)$ has one common virtual edge with skeleton $(v)$, which is called as a virtual edge of $v$. For details, see [2].

The second step is the reduction. The reduction process takes the SPQR-tree of a biconnected graph, rooted at the center, based on the following lemma.

Lemma 1. [1] The center of the SPQR-tree is fixed by a planar automorphism group of a biconnected planar graph.

The reduction process proceeds the SPQR-tree from the leaf nodes to the center level by level, computing labels. The labels are a pair of integers, and boolean values that capture some information of the planar automorphisms of the leaf nodes. First it computes the labels for the leaf nodes. Then it labels the corresponding virtual edge in the parent node and delete each leaf node. The reduction process stops when it reaches the root.

The reduction process clearly does not decrease the planar automorphism group of the original graph. This is not enough; we need to also ensure that the planar automorphism group is not increased by reduction. This is the role of the labels. As a leaf $v$ is deleted, the algorithm labels the virtual edge $e$ of $v$ in skeleton $(u)$ where $u$ is a parent of $v$. Roughly speaking, the labels encode information about the deleted leaf to ensure that every planar automorphism of the labeled reduced graph extends to a planar automorphism of the original graph.

The last step is to compute a maximum size planar automorphism group at the center using the information encoded on the labels.

\subsection{The Labels and Labeling Algorithms}

Standard Labels. Let $v$ be an internal node of $T_{1}$. We say that a virtual edge $e$ of skeleton $(v)$ is a parent (child) virtual edge if $e$ corresponds to a virtual edge of $u$ which is a parent (child) node of $v$. We define a parent separation pair $s=\left(s_{1}, s_{2}\right)$ of $v$ as the two endpoints of a parent virtual edge $e$.

When we compute the labels of $v$, we need to delete the parent virtual edge $e$ from skeleton $(v)$. We denote the resulting graph by skeleton ${ }^{-}(v)$. The union of 
the graphs skeleton ${ }^{-}(u)$ for all descendants $u$ of $v$, including $v$ itself, is denoted by $G^{+}(v)$.

Suppose that nodes $v_{1}, v_{2}, \ldots v_{k}$ of the SPQR-tree $T_{1}$ are deleted at one iteration of the reduction process. These nodes correspond to virtual edges $e_{1}, e_{2}, \ldots, e_{k}$ in the level above the current level. For each $v$, we need to compute the following standard labels.

1. isomorphism code: a pair $I s o(v)$ of integers.

2. axial codes:

(a) $A_{\text {swap }}(v)$ : a boolean label indicating whether $G^{+}(v)$ has an axial symmetry that swaps the parent separation pair.

(b) $A_{f i x}(v)$ : a boolean label indicating whether $G^{+}(v)$ has an axial symmetry that fixes the parent separation pair.

3. rotation code: a boolean label $\operatorname{Rot}(v)$ indicating whether $G^{+}(v)$ has a rotational symmetry of 180 degrees that swaps the parent separation pair.

Note that we need these labels when the virtual edge is fixed by a planar automorphism of the parent node. Further we need to define special labels, which are motivated by the special case below and plays important role to give a linear time algorithm.

Special Case. The aim of labeling is to encode information about planar automorphisms of the skeleton ${ }^{-}(v)$ of the non-root node $v$ of the SPQR tree in the parent virtual edges. In this way we can find planar automorphisms of the whole graph by finding planar automorphisms of the labeled skeleton $(c)$ of the root node $c$. This strategy has a simple flaw: the edges may not model the topological properties of the skeleton ${ }^{-}(v)$ correctly. In particular, while skeleton $^{-}(v)$ of a child node $v$ can enclose skeleton $(u)$ of a parent node $u$ (see Figure $1(\mathrm{a})$ ), the child virtual edge $e$ in the skeleton $(u)$ of parent node $u$ (see Figure 1(b)) cannot enclose skeleton $(u)$, since it is purely a one-dimensional curve. An embedding in which skeleton $(u)$ is on an inside face of $G^{+}(v)$, where $u$ is the parent of $v$, is called an enclosing composition.

Figure 2(a) shows an example of a drawing constructed by an enclosing composition; this shows the maximum number of symmetries of a graph, two rotational and two axial symmetries. Figure 2(b) shows the SPQR tree of the graph. Here skeleton $(c)$ of the root node $c$ of the SPQR tree is enclosed by the skeleton $(v)$ of its child node $v$. The enclosing child node may be in turn enclosed by one of its children, again fixed setwise. Note that the graph can be

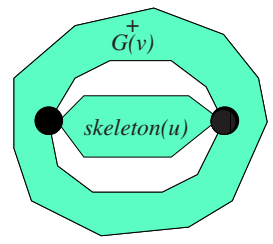

(a)

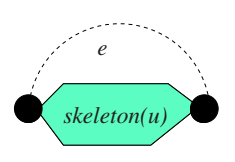

(b)

Fig. 1. An enclosing composition: $v$ is a child of $u$. 


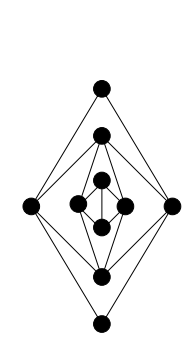

(a)

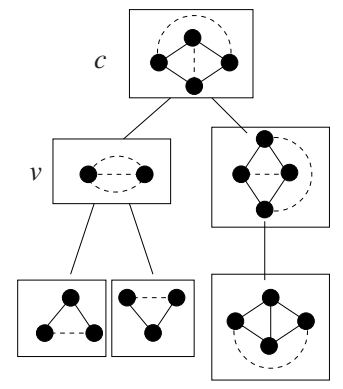

(b)

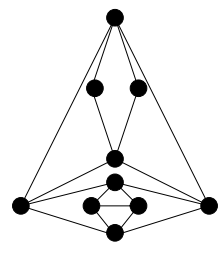

(c)

Fig. 2. The special (enclosing) case.

drawn without enclosing nodes, based on skeleton $(c)$, as in Figure 2(c); but this displays less symmetry, only one axial symmetry.

Special Labels. To take care of special cases such as that illustrated in Figure 2, we define special labels. The most important property of this special case is that the whole planar automorphism group fixes two faces of $G^{+}(v)$ : the outside face, and the internal face containing the separation pair. Thus we define the following labels.

1. special axial codes:

(a) $A_{\text {swap }}^{*}(v)$ : a boolean label indicating whether $G^{+}(v)$ has an axial symmetry that swaps the parent separation pair and with the parent separation pair on an inside face.

(b) $A_{f i x}^{*}(v)$ : a boolean label indicating whether $G^{+}(v)$ has an axial symmetry that fixes the parent separation pair and with the parent separation pair on an inside face.

2. special rotation code: a boolean label $\operatorname{Rot}^{*}(v)$ indicating whether $G^{+}(v)$ has a rotational symmetry that swaps the parent separation pair and with the parent separation pair on an inside face.

In fact, it is enough to require that the planar automorphism fixes two faces: one incident to the parent separation pair, and the other not incident to the parent separation pair, as described in the following lemma.

Lemma 2. Suppose that $D$ is a drawing of a planar graph $G$ and $u$ and $w$ are vertices of $G$ that share a face in $D$. Suppose that $D$ displays an axial planar automorphism $\phi$ that fixes $\{u, w\}$. Then $\phi$ fixes at least one face not incident to $u$ and $w$ if and only if there is a drawing $D^{\prime}$ of $G$ that displays $\phi$, with $u$ and $w$ not on the outside face.

Lemma 2 implies, for example, that $A_{\text {swap }}^{*}(v)=$ true if and only if $G^{+}(v)$ has an axial planar automorphism that swaps the parent separation pair, fixes a face incident to the separation pair, and fixes at least one other face. 
Computation of an Isomorphism Code. The isomorphism code Iso(v) consists of a pair of integers, because the skeleton $(v)$ has an orientation with respect to the parent separation pair. The isomorphism code can be computed in linear time using a planar graph isomorphism algorithm [10]. For details, see [7].

Computation of Axial Codes. An axial symmetry either swaps the parent separation pair or fixes the parent separation pair. First we describe an algorithm for $A_{\text {swap }}(v)$.

Note that the axial symmetry should respect the isomorphism code of the child virtual edge. Further, if the axial symmetry fixes a child virtual edge, then we need to test its label. Further, from Lemma $2, A_{\text {swap }}^{*}(v)$ is true if and only if $A_{\text {swap }}(v)$ is true and the axial symmetry of skeleton $^{-}(v)$ can be extended to an axial symmetry of $G^{+}(v)$ that fixes more than one face. Thus, we can compute both $A_{\text {swap }}(v)$ and $A_{\text {swap }}^{*}(v)$ as follows.

\section{Algorithm Compute_Axial_Code_Swap}

1. Test whether skeleton ${ }^{-}(v)$ has an axial symmetry $\alpha$ which swaps the parent separation pair and respects the isomorphism codes of child virtual edges.

2. If $\alpha$ exists, then

(a) For each child virtual edge $e_{j}$ that is fixed by $\alpha$, check the followings:

i. if $\alpha$ fixes the endpoints of $e_{j}$, then $A_{f i x}\left(v_{j}\right)=\operatorname{true}$.

ii. if $\alpha$ swaps the endpoints of $e_{j}$, then $A_{\text {swap }}\left(v_{j}\right)=$ true.

(b) If one of these properties fails, then $A_{\text {swap }}(v):=$ false; else $A_{\text {swap }}(v):=$ true.

else $A_{\text {swap }}(v):=$ false.

3. If $A_{\text {swap }}(v)=$ false then $A_{\text {swap }}^{*}(v):=$ false;

else if either

$-\alpha$ fixes more than one face, or

- $\alpha$ swaps the endpoints of a child virtual edge $f$ such that $A_{\text {swap }}^{*}(f)=$ true, or

- $\alpha$ fixes the endpoints of a child virtual edge $f$ such that $A_{f i x}^{*}(f)=$ true, then $A_{\text {swap }}^{*}(v):=$ true; else $A_{\text {swap }}^{*}(v):=$ false.

Algorithms for computing $A_{f i x}(v)$ and $\operatorname{Rot}(v)$ are similar to the algorithm for computing $A_{\text {swap }}(v)$. Note that the labeling algorithm correctly computes labels and runs in linear time. For proofs, see [7].

When $v$ is a $P$-node, we use similar algorithms to the case of parallel compositions in series parallel digraphs [5]. When $v$ is an $S$-node, we use similar algorithms to the case of series compositions in series parallel digraphs [5]. For details, see [7].

\subsection{Computing a Maximum Size Planar Automorphism Group at the Center}

The center of the SPQR-tree may be a node or an edge. If the center is a node $c$, then we can further divide into three cases by its type. If $c$ is a $R$-node, then we use the triconnected case [6] to compute a maximum size planar automorphism 
group. If $c$ is a $P$-node, then we use a similar algorithm to the case of a parallel composition in series parallel digraphs (see $[5,7]$ ). If $c$ is an $S$-node, then we can use the algorithm for outerplanar graphs [11].

However, there may exist some other node $v$ which is fixed by a planar automorphism group as in the special case. Thus to find a maximum size planar automorphism group at the center $c$, we consider both cases and then find the maximum. Here we need an algorithm to find a child node which gives the best result for the enclosing composition. This can be done in linear time by using generators. For details, see [7].

If the center is an edge, then we find the maximum among three cases: parallel composition, reduction composition and enclosing composition. Parallel composition means that we construct a drawing with two labeled edges such as a parallel composition in series parallel digraphs. Reduction composition means that we compute labels of one node $u$ and then delete $u$ by labeling the corresponding virtual edge $e$ of the other node $v$. Then we compute a planar automorphism group at $v$ using the center node case. Enclosing composition means that we construct a drawing such as the special case. Each of these cases can be computed in linear time, see [7].

\section{The One-Connected Case}

The algorithm for computing a maximum size planar automorphism group of one-connected planar graph has a similar flavor to the biconnected case: we use reduction approach. We also use algorithm Biconnected_Planar as a subroutine. The reduction process is similar to the biconnected case. In this case we take the block-cut vertex tree (BC-tree) and then compute labels at each leaf node (block or cut vertex). However, the labels are different.

\subsection{The Labels and Labeling Algorithms}

We need two types of labels: isomorphism code and axial code. However, these are further divided into the case of a cut vertex or a block. Let $B$ represent a block and $C$ represent a cut vertex.

1. isomorphism code : an integer $I s o_{B}(v)\left(\right.$ or $\left.I s o_{C}(v)\right)$.

2. axial code : an integer $A_{B}(v)$ (or $A_{C}(v)$ ) indicating whether $B$ (or $C$ ) has an axial symmetry which fixes the parent node.

Note that we need these labels when the block or cut vertex is fixed by a planar automorphism of the parent node.

Computation of an Isomorphism Code of a Block. Suppose that $B_{1}, B_{2}$, $\ldots, B_{m}$ are the blocks on the lowest level and $p_{1}, p_{2}, \ldots, p_{m}$ are the parent cut vertices for the blocks. We compute isomorphism code $I s o_{B}\left(v_{i}\right)$ for each $B_{i}$ using a planar graph isomorphism algorithm which takes linear time [10]. Note that the isomorphism should respect the isomorphism code of the child cut vertex. We now describe the algorithm. 


\section{Algorithm Compute_Iso_B}

for each $B_{i}, i=1,2, \ldots, m$,

if there is an isomorphism $\alpha$ between $B_{i}$ and $B_{j}$ such that

(a) $\alpha\left(p_{i}\right)=p_{j}$, and

(b) for each cut vertex $c_{k}$ of $B_{i}$,

i. $\alpha\left(c_{k}\right)$ is a cut vertex, and

ii. $\operatorname{Iso}_{C}\left(c_{k}\right)=\operatorname{Iso}_{C}\left(\alpha\left(c_{k}\right)\right)$.

then assign isomorphism codes such that $I s o_{B}\left(v_{i}\right)=I s o_{B}\left(v_{j}\right)$.

Computation of an Axial Code of a Block. The label $A_{B}(v)$ represents whether the block $B$ has an axial symmetry which fixes the parent cut vertex $p$. Let $c_{1}, c_{2}, \ldots, c_{k}$ be the child cut vertices of $B$. In fact, the algorithm computes a ternary value for $A_{B}(v)$. The interpretation of $A_{B}(v)$ is:

1. $A_{B}(v)=1$ if $G^{+}(v)$ has an axial symmetry that fixes $p$ and one face incident to $p$.

2. $A_{B}(v)=2$ if $G^{+}(v)$ has an axial symmetry which fixes $p$ and two faces incident to $p$.

3. $A_{B}(v)=3$ if $G^{+}(v)$ has no axial symmetry that fixes $p$ and a face incident to $p$.

First we find an axial symmetry $\alpha$ of $B$ which fixes the parent cut vertex using Biconnected_Planar. Then we check whether each fixed child cut vertex $c_{j}$ preserves the axial symmetry. For this purpose, we need some information about the axial code $A_{C}(v)$. The interpretation of values of $A_{C}(v)$ is:

1. $A_{C}(v)=0$ if $G^{+}(v)$ has an axial symmetry which does not fix any $G^{+}\left(v_{i}\right)$ for any $i$.

2. $A_{C}(v)=1$ if

(a) every axial symmetry of $G^{+}(v)$ fixes at least one $G^{+}\left(v_{i}\right)$, and

(b) there is an axial symmetry $\alpha$ such that if $\alpha$ fixes $G^{+}\left(v_{i}\right)$ then $A_{B}\left(v_{i}\right)<3$, and there is at most one $j$ such that $\alpha$ fixes $G^{+}\left(v_{j}\right)$ and $A_{B}\left(v_{j}\right)=1$.

3. $A_{C}(v)=2$ if

(a) every axial symmetry of $G^{+}(v)$ fixes at least two $G^{+}\left(v_{i}\right)$ for which $A_{B}\left(v_{i}\right)=1$, and

(b) there is an axial symmetry $\alpha$ such that if $\alpha$ fixes $G^{+}\left(v_{i}\right)$ then $A_{B}\left(v_{i}\right)<$ 3 , and there are at most two indices $j$ such that $\alpha$ fixes $G^{+}\left(v_{j}\right)$ and $A_{B}\left(v_{j}\right)=1$.

4. $A_{C}(v)=3$ otherwise.

Finally we assign the value, depending on the fixed faces which are adjacent to $p$.

We now state the algorithm.

Algorithm Compute_Axial_B

Apply Biconnected_Planar [7] to the labelled graph $B$; if $B$ has an axial planar automorphism $\alpha$ such that

(a) $\alpha$ fixes $p$ and respects the isomorphism partition labels on $B$;

(b) For each child cut vertex $c_{j}$ fixed by $\alpha$, the number of faces incident to

$c_{j}$ and fixed by $\alpha$ is at least as large as $A_{C}\left(c_{j}\right)$.

then $A_{B}(v):=$ the number of faces fixed by $\alpha$ and incident to $p$.

else $A_{B}(v):=3$. 
Computation of an Isomorphism Code of a Cut Vertex. Suppose that $c_{1}, c_{2}, \ldots, c_{k}$ are the cut vertices on the lowest level. We compute $\operatorname{Iso}_{C}\left(c_{i}\right)$ for each $c_{i}, i=1,2, \ldots, k$, which represents an isomorphism code of $c_{i}$. More specifically, $\operatorname{Iso}_{C}\left(c_{i}\right)=I s o_{C}\left(c_{j}\right)$ if and only if the subgraph which is rooted at $c_{i}$ is isomorphic to the subgraph which is rooted at $c_{j}$. We now state the algorithm.

\section{Algorithm Compute_Iso_C}

1. For each $c_{i}$ :

(a) Let $B_{i 1}, B_{i 2}, \ldots, B_{i m}$ be the child blocks of $c_{i}$.

(b) $s\left(c_{i}\right):=\left(I \operatorname{Iso}_{B}\left(B_{i 1}\right), \operatorname{Iso}_{B}\left(B_{i 2}\right), \ldots, I s o_{B}\left(B_{i m}\right)\right)$.

(c) Sort $s\left(c_{i}\right)$.

2. Let $Q$ be the list of $s\left(c_{i}\right), i=1,2, \ldots, k$.

3. Sort $Q$ lexicographically.

4. For each $c_{i}$, compute $I \operatorname{so}_{C}\left(c_{i}\right)$ as follows: Assign the integer 1 to $c_{i}$ whose $s\left(c_{i}\right)$ is the first distinct tuple of the sorted sequence $Q$. Assign the integer 2 to $c_{j}$ whose $s\left(c_{j}\right)$ is the second distinct tuple, and so on.

Computation of an Axial Code of a Cut Vertex. The label $A_{C}(v)$ represents whether a cut vertex $c$ has an axial symmetry which fixes the parent block. Let $B_{p}$ be the parent block of $v$ and $B_{1}, B_{2}, \ldots, B_{m}$ be the child blocks of $v$. Suppose that $\alpha$ is an axial symmetry which fixes $B_{p}$. We use $A_{C}(v)$ to decide whether $c$ preserves $\alpha$ of $B_{p}$. More specifically, this indicates that whether $B_{1}, B_{2}, \ldots, B_{m}$ can be attached to $c$, preserving $\alpha$.

To compute $A_{C}(v)$, we use $A_{B}\left(B_{j}\right)$. The label $A_{B}\left(B_{j}\right)$ indicates that whether $B_{j}$ has an axial symmetry which fixes $c$. Further, it indicates that whether there is a fixed face adjacent to $c$. We now state the algorithm.

\section{Algorithm Compute_Axial_C}

1. Partition $B_{1}, B_{2}, \ldots, B_{m}$ into isomorphism classes $P_{\ell}$ using $I \operatorname{so}_{B}\left(B_{j}\right)$, and compute the size $s_{\ell}$ of each isomorphism class $P_{\ell}$.

2. If all $s_{\ell}$ are even, then $A_{C}(v):=0$; exit.

3. If there is an $s_{\ell}$ that is odd and $A_{B}\left(v_{j}\right)=3$ for each $j \in P_{\ell}$ then $A_{C}(v):=3$; exit.

4. Let $f$ be the number of odd $s_{\ell}$ such that if $j \in P_{\ell}$ then $A_{B}\left(v_{j}\right)=1$.

(a) If $f>2$ then $A_{C}(v):=3$.

(b) If $f=2$ then $A_{C}(v):=2$.

(c) If $f=1$ then $A_{C}(v):=1$.

Note that all the labeling algorithms correctly compute labels and run in linear time, see [8].

\subsection{Computing a Maximum Size Planar Automorphism Group at the Center}

We can compute a maximum size planar automorphism group of the whole graph by computing a maximum size planar automorphism group at the labeled center, based on the following lemma. 
Lemma 3. The center of the BC-tree is fixed by a planar automorphism group of a one-connected planar graph.

The algorithm can be divided into two cases, since the center of the BC tree may be a block or a cut vertex. Roughly speaking, if the center is a block $B$, then we use algorithm Biconnected_Planar in Section 3. If the center is a cut vertex $c$, then we use a similar method that was used in the case of trees [11].

However, the algorithm is not as simple as this, mainly because there are some special cases. Namely, there may exist a fixed cut vertex when the center is a block, and there may exist a fixed block when the center is a cut vertex. To illustrate the special cases, see the graph Figure 3, with its BC tree. The center of the $\mathrm{BC}$ tree is a block $B$ with 5 cut vertices. The symmetries of the drawing of this block fix the cut vertex $c$ with 4 children in the $\mathrm{BC}$ tree. Maximizing symmetry for the whole graph is not merely a matter of the reduction process plus maximizing symmetry of $B$; we must also arrange the children of the fixed cut vertex in a symmetrical way. Essentially this means merging the symmetries of $G^{+}(c)$ with the symmetries of $G^{+}(B)$. Similar case can happen when the center is a cut vertex. Thus to compute the maximum, we need to consider these special cases. Again, we need an algorithm to find the child vertex of the center which can gives the maximum. However, this can be done in linear time, see [8].

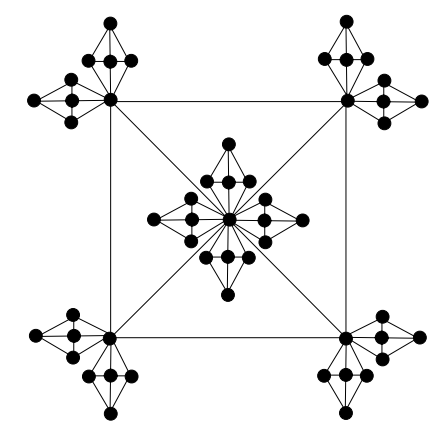

(a)

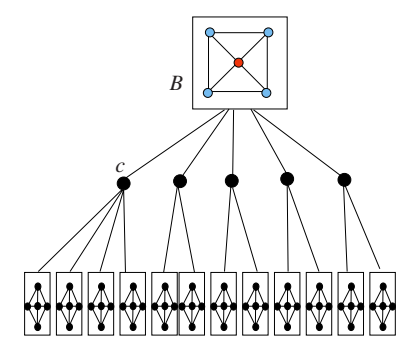

(b)

Fig. 3. Special case.

\section{The Drawing Algorithms}

The algorithms presented in the preceding sections take a biconnected planar graph and one-connected planar graph as input and has two outputs: a planar automorphism group of maximum size, and an embedding of the graph. In this section, we show how to use this information to construct a straight line symmetric drawing of the graph.

Given an embedding of a biconnected planar graph, we use "augmentation": we increase the connectivity by adding new edges and new vertices to make it triconnected, while preserving the planar automorphism group. Then we can 
apply the algorithm for constructing symmetric drawings of triconnected planar graphs with straight-line edges to construct a symmetric drawing [6]. The algorithm runs in linear time [6]. Finally we delete the added edges and vertices.

Given an embedding of a one-connected planar graph, we use "attachment": first, we draw each block using the algorithm for constructing symmetric drawings of biconnected planar graphs with straight-line edges to construct a symmetric drawing. Then we attach each drawing at each cut vertex, preserving planarity. Clearly, this algorithm runs in linear time.

\section{References}

1. L. Babai, Automorphism Groups, Isomorphism, and Reconstruction, Chapter 27 of Handbook of Combinatorics Volume 2 (ed. Graham, Groetschel and Lovasz), Elsevier Science, 1995.

2. G. Di Battista and R. Tamassia, On-Line Planarity Testing, SIAM Journal on Computing 25(5), pp. 956-997, 1996.

3. P. Eades and X. Lin, Spring Algorithms and Symmetries, Theoretical Computer Science 240, pp. 379-405, 2000.

4. S. Hong, P. Eades and S. Lee, An Algorithm for Finding Geometric Automorphisms in Planar Graphs, Algorithms and Computation, Proc. of ISAAC 98, Lecture Notes in Computer Science 1533, pp. 277-286, Springer Verlag, 1998.

5. S. Hong, P. Eades and S. Lee, Drawing Series Parallel Digraphs Symmetrically, Computational Geometry: Theory and Applicatons 17(3-4), pp. 165-188, 2000.

6. S. Hong, B. McKay and P. Eades, Symmetric Drawings of Triconnected Planar Graphs, Proc. of SODA 2002, pp. 356-365, 2002.

7. S. Hong and P. Eades, Drawing Planar Graphs Symmetrically II: Biconnected Graphs, Technical Report CS-IVG-2001-01, Basser Department of Computer Science, The University of Sydney, 2001, Submitted.

8. S. Hong and P. Eades, Drawing Planar Graphs Symmetrically III: One-connected Graphs, Technical Report CS-IVG-2001-02, Basser Department of Computer Science, The University of Sydney, 2001, Submitted.

9. S. Hong and P. Eades, Symmetric Layout of Disconnected Graphs, Algorithms and Computation, Proc. of ISAAC 2003, Lecture Notes in Computer Science 2906, pp. 405-414, Springer Verlag, 2003.

10. J. E. Hopcroft and J. K. Wong, Linear Time Algorithm for Isomorphism of Planar Graphs, Proc. of the Sixth Annual ACM Symposium on Theory of Computing, pp. 172-184, 1974.

11. J. Manning, Geometric Symmetry in Graphs, Ph.D. Thesis, Purdue Univ., 1990. 